\title{
Optical Rotatory Dispersion of Liquid Crystal Solutions of a Triple-Helical Polysaccharide Schizophyllan
}

\author{
Kazuo Van, Toshiaki Asakawa, and Akio Teramoto \\ Department of Macromolecular Science, Osaka University, \\ Toyonaka, Osaka 560, Japan
}

(Received November 4, 1983)

\begin{abstract}
When confined in a thin parallel cell, a liquid crystal solution of a polysaccharide schizophyllan formed a planar texture and showed very strong optical rotatory power. The wavelength dependence of the optical rotatory power was represented by a simple equation of the Drude type and differed from that of an isotropic solution which obeyed a Moffitt-Yang type equation. The data at longer wavelengths were consistent with the prediction by the de Vries theory of cholesterics, yielding a layer birefringence approximately proportional to the polymer concentration. The intrinsic birefringence of a schizophyllan triple helix was estimated to be about 0.02 , which is much smaller than that of xanthan and the birefringence of crystalline cellulose. The difference in birefringence was ascribed to the difference in chain conformation among these glucans. An abrupt change in ORD behavior occurred when an isotropic solution was cooled down to a temperature close to the isotropic-biphasic boundary temperature, indicating the occurrence of a pretransition from isotropic to cholesteric phases.
\end{abstract}

KEY WORDS Optical Rotatory Dispersion / Cholesteric Liquid Crystal /

Polysaccharide / Schizophyllan / Drude Equation / Rodlike Polymer /

Birefringence / Phase Equilibrium / Planar Structure / Cholesteric Pitch /

In our recent publications $s^{1,2}$ it was shown that aqueous solutions of a triple helical polysaccharide schizophyllan form a cholesteric mesophase above a certain critical polymer concentration. One striking optical property of the cholesteric mesophase is an extraordinary optical rotation for the light propagating along the cholesteric axis. ${ }^{3-6}$ Therefore, it was natural to expect similar optical rotation behavior for our schizophyllan liquid crystals. In the previous study, ${ }^{1}$ an optical rotation measurement on liquid crystal solutions was attempted without success; it should be noted, however, that a remarkable enhancement in optical rotatory power was observed when the temperature of an isotropic solution was lowered toward the isotropic-biphasic phase boundary. Another such attempt was made in the present study. The experimental results described below show that the above expec- tation holds true, confirming that the system under examination is a cholesteric mesophase. An enhancement in optical rotatory power near the isotropic-biphasic phase boundary, similar to that found by Patel and DuPré ${ }^{7}$ on polypeptide solutions, is also described.

\section{EXPERIMENTAL}

A schizophyllan sample V-1 of $c a .400,000$ molecular weight supplied by Taito Co. was used except in a few experiments, in which another sample U-1 of 170,000 molecular weight also from Taito was studied. These samples were prepared from native schizophyllan by a method described elsewhere. ${ }^{8}$ It was expected from our previous studies ${ }^{1,2,9}$ that the former sample might become fully cholesteric at concentrations above $13 \mathrm{wt} \%$, whereas the critical concentration for the 
latter might be around $20 \mathrm{wt} \%$.

Polysaccharide solutions were prepared by mixing appropriate amounts of the sample and water in a small weighing bottle and the concentrations (expressed in terms of weight fraction $w_{2}$ or volume fraction $\phi$ of the polymer) were determined gravimetrically; $\phi$ was calculated from $w_{2}$ by $\phi=w_{2} v /\left[w_{2} v+\left(1-w_{2}\right) /\right.$ $\rho$ ] where $v$ is the partial specific volume of schizophyllan, $0.619 \mathrm{~cm}^{3} \mathrm{~g}^{-1}$, and $\rho$, the density of water at $25^{\circ} \mathrm{C}$. Optical rotation measurements were made at about $20^{\circ} \mathrm{C}$ using a JASCO ORD/UV-5 spectropolarimeter in the range of wavelength between 220 and $650 \mathrm{~nm}$; glass cells were used down to $300 \mathrm{~nm}$, and quartz ones down to $220 \mathrm{~nm}$. Cells differing in thickness were constructed as follows. A thin plastic film was sandwiched between two slide glass plates $(c a .1 \times 10 \times 40 \mathrm{~mm})$ slightly wider than the film. The longer edges of the plates were sealed with quick adhesive. After about one hour, the film was pulled out, leaving a thin cavity to be used for the solution compartment. The cell thickness $d$ was adjusted by changing the film thickness. Thicker cells were constructed more easily by binding two plates with strips of Scotch tape; $d$ was controlled by the number of strips placed. The cells thus constructed, whether empty or filled with water, showed negligible optical rotation and the thickness of each cell was determined within an accuracy of $\pm 2 \mu \mathrm{m}$. The cells were filled with solutions under reduced pressure, sealed at the ends with an adhesive material and placed in an air bath thermostatted at $25^{\circ} \mathrm{C}$ except at the time of physical measurements. The other experimental details were the same as described previously.,

\section{RESULTS AND DISCUSSION}

\section{Planar Structure and Extraordinary Optical Rotation}

Figure 1 shows photomicrographs between crossed polars for a $14.34 \mathrm{wt} \%$ solution in parallel glass cells of different thicknesses.
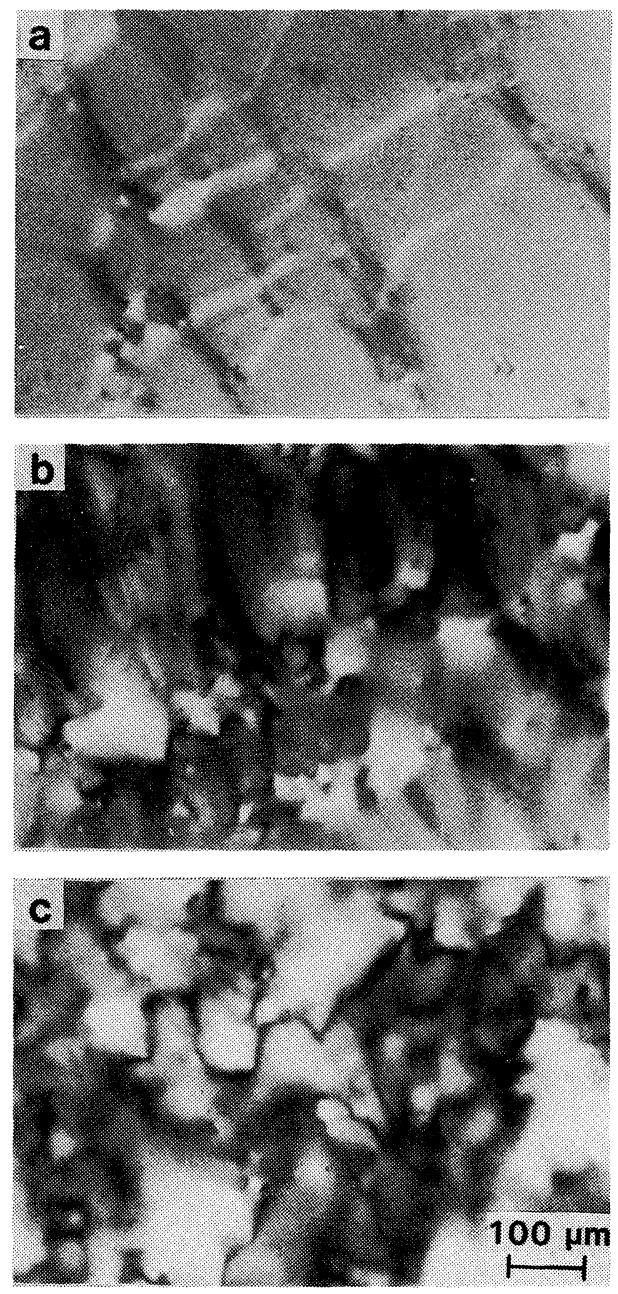

Figure 1. Photomicrographs between crossed polars of a $14.34 \mathrm{wt} \%$ solution of schizophyllan in water placed in parallel glass cells of different thicknesses $d$. (a) planar texture, $d=81 \mu \mathrm{m}$; (b) and (c), multi-domain structure, $d=158 \mu \mathrm{m}$ and $315 \mu \mathrm{m}$, respectively.

When contained in a cell with $d$ smaller than $100 \mu \mathrm{m}$, the solution showed no birefringence and appeared almost dark between crossed polars; Figure 1a is a typical photograph, where white streaks (or threads) can be seen in the dark background.

In most preparations, these streaks consisted of bundles of alternating bright and dark parallel lines, i.e., fingerprint patterns, running parallel to the streaks. The streaks parallel to a 
polar were almost invisible, but those inclined by $45^{\circ}$ from the polar were brightest; those normal to the brightest streaks were darker than the background. Observation under a polarizing microscope equipped with a quartz wedge revealed that the direction of a larger refractive index was the same as the parallel lines. We thus concluded that a pile of cholesteric layers was being viewed in the direction normal to the layers. Figure la shows a planar texture of the cholesteric mesophase. It is not likely that the dark area in Figure 1a is due to a homeotropic texture because this preparation had extraordinary optical rotation, as will be mentioned below.

The appearance of the planar texture may be understood if it is assumed that, due to the restraint exerted by the cell walls, the layers of the cholesteric phase are forced to align themselves parallel to the cell walls when confined in a thin cell. The bright streaks are the places where the layers are oriented normal to the cell walls. As the cell thickness increases, restraint by the cell walls should diminish enough so that the cholesteric layers are no longer forced to be oriented parallel to the walls. Indeed, for cells thicker than $130 \mu \mathrm{m}$, a multi-domain structure such as any one of those seen in Figures $1 b$ and $1 c$, instead of the planar texture, predominantly filled the entire area. Such a strucrure may be an assembly of small cholesteric domains aligned in various directions.

Liquid crystal solutions of schizophyllan were found to have extraordinary optical rotation in comparison with isotropic solutions and this optical rotation behavior varied with the thickness of the preparation examined. For preparations thinner than $100 \mu \mathrm{m}$, the optical rotation increased gradually during a period of about one month after having filled the cells and then appeared to reach an equilibrium state. On the other hand, change in optical rotation was quite irregular and less reproducible for thicker preparations. We consider that after having filled in a thinner cell, the cholesteric structure, once destroyed by shear stress during filling, is reconstructed and then gradually fits into the narrow space between the cell walls. This reconstruction of microscopic domains may be a relatively fast process, but fitting into the container may be

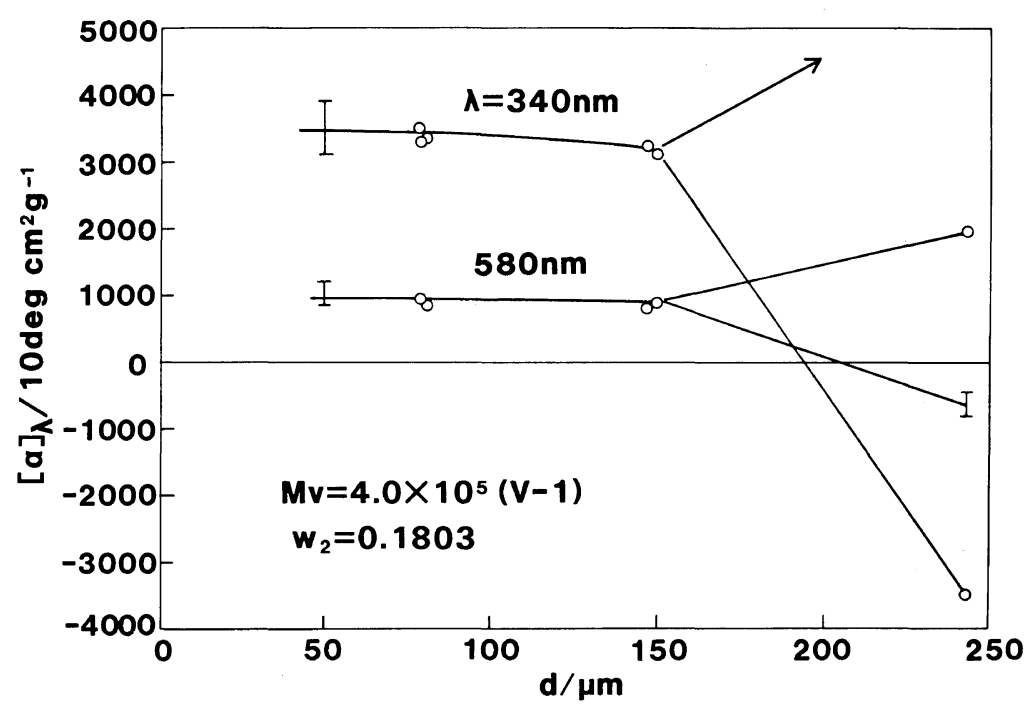

Figure 2. Dependence of optical rotation $[\alpha]_{\lambda}$ on cell thickness $d$ at the indicated wavelengths $\lambda$, $w_{2}=0.1803$. 
much slower because of the high viscosity of the solution. This explains the gradual increase in optical rotation for thinner preparations.

Figure 2 shows the $d$ (cell thickness) dependence of specific rotation $[\alpha]_{\lambda}$ of the 18.03 wt $\%$ solution at fixed wavelengths. Most data were taken more than one month after the filling and thus pertain to the equilibrium state. It can be seen that $[\alpha]_{\lambda}$ remains almost constant at smaller $d$ where a planar structure is observable; the data were relatively reproducible in this $d$ range. But for $d$ greater than $130 \mu \mathrm{m},[\alpha]_{\lambda}$ was much less reproducible and did not reach an equilibrium value. As seen in Figure 2, one thick preparation showed disimilar optical rotation behavior in that the sign as well as magnitude of the rotation changed depending on whether it faced the light beam or not. Some thick preparations showed much greater optical rotation. At present we suspect that the multi-domain structure may be responsible for the anomaly found in the thick preparations. The scattering of data, either for the thinner or thicker preparations, may be due partly to experimental difficulty in handling the viscous solutions. No wall effects on optical rotation such as those noted by Robinson $^{3}$ and Uematsu and Uematsu ${ }^{10}$ were found in our schizophyllan liquid crystals.

For a thin preparation with $w_{2}=0.1434$, the optical rotation remained essentially constant between 15 and $35^{\circ} \mathrm{C}$, but increased abruptly at about $9^{\circ} \mathrm{C}$ to another constant value. The reason for this jump is not understood. In the discussion to follow, attention will be directed to the normal optical rotation behavior of thinner preparations at about $20^{\circ} \mathrm{C}$.

Figure 3 compares the optical rotatory dispersion (ORD) curve of a liquid crystal solution $\left(w_{2}=0.1434\right)$ with that of an isotropic solution $\left(w_{2}=0.0233\right)$. It can be seen that the two curves distinctly differ both in magnitude and in shape. This strong optical rotatory power of the liquid crystal solution gives additional support to the previous conclusion ${ }^{1}$ that our schizophyllan liquid crystal is cholesteric.

Figure 4 shows ORD curves of cholesteric

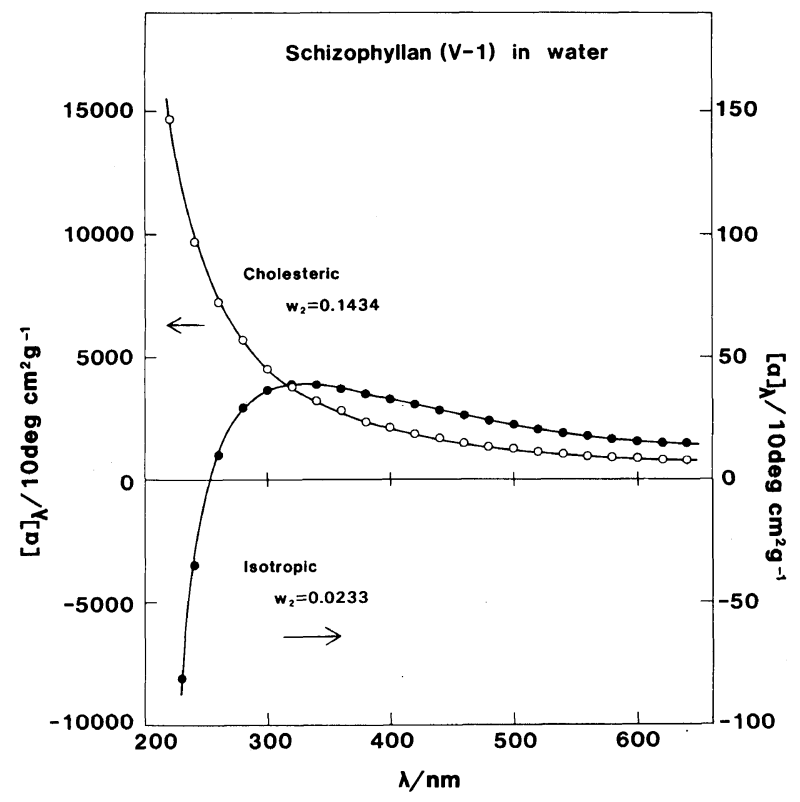

Figure 3. Comparison of ORD curves at $25^{\circ} \mathrm{C}$ between an isotropic solution $\left(w_{2}=0.0233\right)$ and a cholesteric solution $\left(w_{2}=0.1434\right)$. 


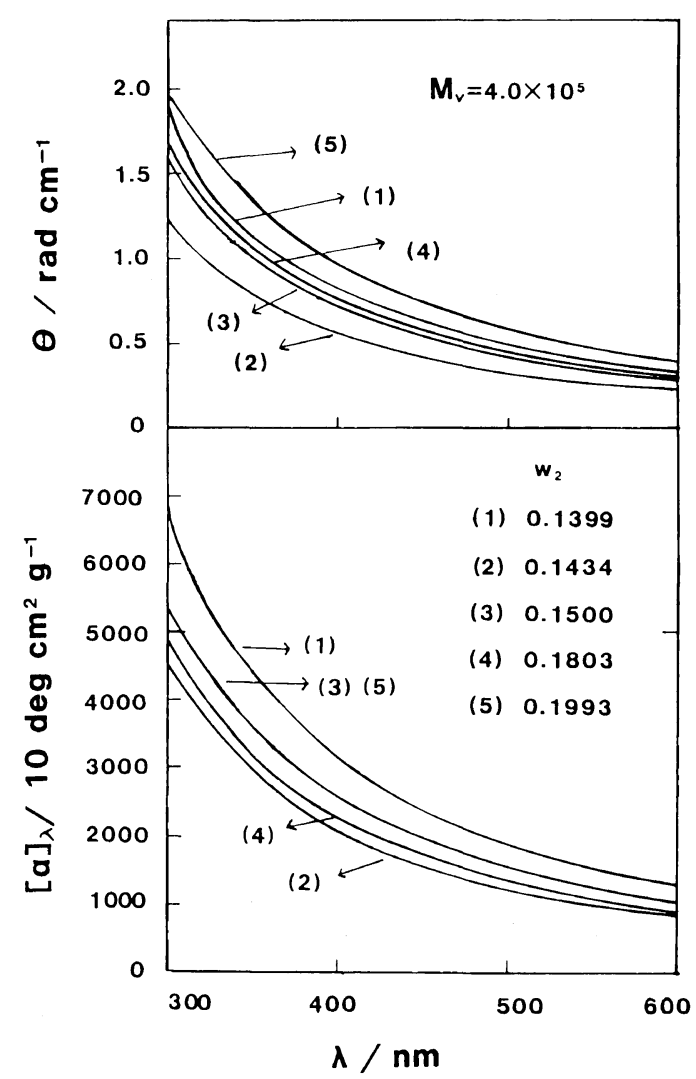

Figure 4. ORD curves of cholesteric solutions with different concentrations.

solutions with different concentrations. It should be noted that neither $\Theta$ nor $[\alpha]_{\lambda}$ varies systematically with the polymer concentration. This is also in contrast to the isotropic behavior that $[\alpha]_{\lambda}$ decreases slowly with increasing concentration: $[\alpha]_{\lambda}$ at $580 \mathrm{~nm}$ decreases from $2.0 \mathrm{deg} \mathrm{cm}^{2} \mathrm{~g}^{-1}$ at infinite dilution to 1.3 $\operatorname{deg} \mathrm{cm}^{2} \mathrm{~g}^{-1}$ at $w_{2}=0.10$.

\section{Analysis by the de Vries Theory}

According to the theory of de Vries, ${ }^{5}$ the optical rotation $\Theta$ for the light beam propagating normal to a cholesteric layer, that is, parallel to the cholesteric axis, varies with the wavelength $\lambda$ of the light in vacuo as

$$
\Theta=\frac{\pi(\Delta n)^{2} P}{4 \lambda^{2}\left(1-\lambda^{2} / \lambda_{0}^{2}\right)}
$$

for $\lambda \gg \Delta n P$, where $\lambda_{0}=n P, P$ the cholesteric pitch and $n$ the average refractive index of the cholesteric. $\Delta n$ is the layer birefringence, i.e., $\Delta n=n_{\|}-n_{\perp}$, with $n_{\|}$and $n_{\perp}$ being the refractive indices of the layers parallel and normal to the director, respectively. For most cholesterics and schizophyllan solutions as well, $\lambda \ll \lambda_{0}$, and hence eq 1 is simplified to give

$$
\Theta=\frac{\pi(\Delta n)^{2} P}{4 \lambda^{2}}
$$

for $\Delta n P \ll \lambda \ll \lambda_{0}$.

The validity of this equation for polymer systems was tested first by Robinson ${ }^{3,4}$ on polypeptide liquid crystals; he determined all the measurable quantities on both sides of the equation and proved the equality. Later, Patel and DuPré ${ }^{7}$ argued against Robinson's conclusion, with their $[\alpha]_{\lambda}$ data inversely proportional to $P$; however they made no direct measurement on $\Delta n$. Recently, Toriumi et al. ${ }^{11}$ reported experimental data supporting the de Vries theory, indicating for a cholesteric solution of poly ( $\gamma$-benzyl L-glutamate) in $m$ cresol that $[\alpha]_{\lambda}$ varied linearly with $\lambda^{-2}$ and that $[\alpha]_{\lambda}$ at a given $\lambda$ was related linearly to $P$ when $P$ changed from positive to negative with raising temperature; under their experimental conditions $\Delta n$ remained essentially constant. Recently, Tseng et al. ${ }^{12}$ showed the validity of eq 1 with data for liquid crystals based on poly(acetoxypropyl) cellulose.

Optical rotation data for thinner preparations of different $w_{2}$ were analyzed by eq 2 . Typical results are displayed in Figure 5, with $\Theta$ plotted against $\lambda^{-2}$. It can be seen that for either preparation, the data points at larger wavelengths fit reasonably well to a straight line, indicating that eq 2 is valid at least within the specified region of the wavelength. The data points at smaller $\lambda$ appear to deviate upward from the straight line. Similar plots were obtained with the data for other preparations, and $\Delta n$ was determined by eq 2 from the slope values of the straight lines together with the $P$ values determined separately. The 


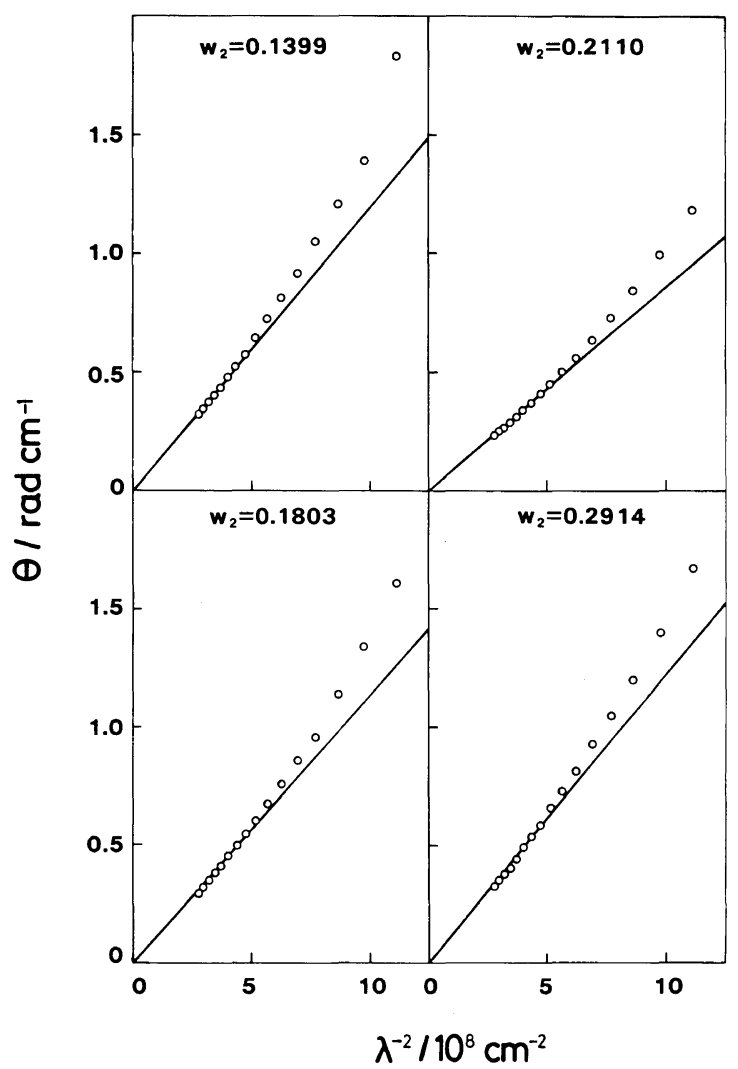

Figure 5. Plots of $\Theta v s . \lambda^{-2}$ for aqueous solutions of V-1 $\left(w_{2}=0.1399,0.1803\right)$ and U-1 $\left(w_{2}=0.2110\right.$, 0.2914).

Table I. Birefringence of the schizophyllan liquid crystal determined from ORD and retardation data

\begin{tabular}{|c|c|c|c|c|}
\hline \multirow{2}{*}{ Samples } & \multirow{2}{*}{$w_{2}$} & \multirow{2}{*}{$P / \mu \mathrm{m}$} & \multirow{2}{*}{$\frac{\mathrm{ORD}}{\Delta n \times 10^{3}}$} & \multirow{2}{*}{$\frac{\text { Retardation }}{\Delta n \times 10^{3}}$} \\
\hline & & & & \\
\hline \multirow[t]{5}{*}{ V-1 } & 0.1399 & $11.2 \pm 0.2$ & $1.1_{6} \pm 0.04$ & - \\
\hline & 0.1434 & $8.0 \pm 0.2$ & $1.1_{5} \pm 0.04$ & $1.2 \pm 0.2$ \\
\hline & 0.1500 & $8.9^{-}$ & $1.2_{2} \pm 0.03$ & - \\
\hline & 0.1803 & 5.9 & $1.5_{6} \pm 0.03$ & - \\
\hline & 0.1993 & $4.1 \pm 0.1$ & $2.1_{0} \pm 0.05$ & $1.8 \pm 0.3$ \\
\hline \multirow[t]{3}{*}{ U-1 } & 0.2110 & $3.4 \pm 0.6$ & $1.7 \pm 0.1$ & - \\
\hline & 0.2490 & $3.4^{-}$ & $2.1_{0} \pm 0.05$ & - \\
\hline & 0.2914 & $2.0_{6}$ & $2.7_{6} \pm 0.03$ & - \\
\hline
\end{tabular}

numerical results obtained are summarized in Table I. According to eq 1 , the positive optical rotation for $\lambda \ll \lambda_{0}$ implies that our schizophyl-

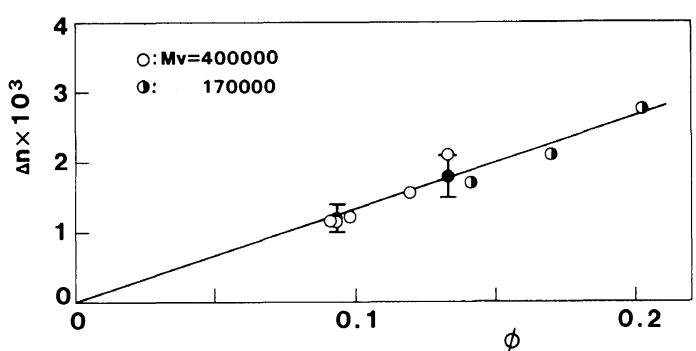

Figure 6. Dependence of the layer birefringence $\Delta n$ on polymer volume fraction $\phi$. Open circles, from ORD data for sample V-1; filled circles, from retardation data for $\mathrm{V}-1$; half-filled circles, from ORD data for U-1.

lan liquid crystal is right-handed.

\section{Birefringence of the Cholesteric Layer}

Figure 6 shows that $\Delta n$ increases approximately linearly with the volume fraction $\phi$ of 
the polymer, yielding an average value of 0.014 for $\Delta n / \phi$. This $\Delta n / \phi$ value may be compared with 0.029 and $0.024-0.038$ for polypeptide liquid crystals reported by Robinson ${ }^{4}$ and DuPré and Lin, ${ }^{13}$ resepctively, and -0.04 $\left(-0.025 \mathrm{~cm}^{3} \mathrm{~g}^{-1}\right)$ by Maret et al. ${ }^{14}$ for xanthan liquid crystals, deduced from either a similar analysis or direct measurement. The absolute values of the $\Delta n / \phi$ are smaller than 0.07 reported for the birefringence of crystalline cellulose, ${ }^{15}$ which consists of $\beta$-1,4-linked D-glucose residues in an almost fully extended conformation. ${ }^{16}$

An independent estimate of $\Delta n$ was attempted in the following way. When observed between crossed polars with a quartz wedge inserted along a streak, the streak exhibited a negative retardation relative to the background which showed no birefringence, judging from the different colors of the two areas. When the wedge was displaced slightly in the direction of increasing retardation, the streak showed the original color of the background. The streak normal to the wedge made a positive contribution to the retardation. Knowing the geometry and birefringence of the wedge, we estimated the change in retardation $\Delta R$ due to this displacement.

If the streak represents a cross-sectional view of the layer structure developing normal to the cell walls all the way from the upper cell wall to the bottom, $\Delta R$ may be related to the birefringence $n_{\|}{ }^{\prime}-n_{\perp}{ }^{\prime}$ of the streak by

$$
\Delta R=\left(n_{\|}{ }^{\prime}-n_{\perp}{ }^{\prime}\right) d
$$

where $n_{\|}{ }^{\prime}$ and $n_{\perp}{ }^{\prime}$ are the average refractive indices parallel and normal to the streak. Since the cholesteric layers are aligned parallel to the streak, $n_{\perp}{ }^{\prime}$ can be equated to $n_{\perp}$. The refractive index parallel to the streak varies periodically between $n_{\|}$and $n_{\perp}$ in the direction normal to the streak, and $n_{\|}{ }^{\prime}$ can be taken as the average of $n_{\|}$and $n_{\perp}$, i.e., $\quad n_{\|}{ }^{\prime}=(1 / 2)\left(n_{\|}+n_{\perp}\right)$. Therefore, we have

$$
\Delta R=d \Delta n / 2
$$

Relatively thin preparations which might possibly develop a layer structure over the entire cell thickness were examined to find appropriate streaks. A number of measurements on such streaks at different places and for different preparations was averaged to obtain $\Delta n$ as a function of $w_{2}$, with only moderate accuracy. The results are shown in Figure 6 by filled circles. The values of $\Delta n$ obtained in the two independent ways are in fair agreement, giving additional support to eq 2. Direct refractometry, as employed by previous investigators, ${ }^{12,13}$ failed to provide meaningful results.

The birefringence of a lyotropic liquid crystal may possibly arise from uniaxially oriented rodlike molecules intrinsically aniostropic in polarizability. Therefore, it should be reasonable to assume that $\Delta n$ is expressed by the product of the intrinsic birefringence $n_{\alpha}-n_{\gamma}$ of the molecule, the number of molecules in unit volume, and the extent to which they are oriented. The last factor may be taken into account by the order parameter $S$ defined by

$$
S=(1 / 2)\left\langle 3 \cos ^{2} \theta-1\right\rangle
$$

where $\theta$ is the angle between the long axis of a molecule and the director and $\langle\cdots\rangle$ means the average over all molecules. It then follows from the above assumption that $\Delta n$ may be expressed as ${ }^{17}$

$$
\Delta n=\left(n_{\alpha}-n_{\gamma}\right) \phi S
$$

At present, no experimental information on either $n_{\alpha}-n_{\gamma}$ or $S$ is available for our schizophyllan liquid crystals. A theoretical consideration on nematic liquid crystals ${ }^{18}$ suggests that $S$ increases from about 0.5 to unity as $\phi$ tends to unity. Thus, the value of $n_{\alpha}-n_{\gamma}$ for the system schizophyllan + water may be larger than 0.014 by a factor not greater than 2, yielding 0.028 as the upper limit; the most probable value may be around 0.02 .

Therefore, we see that even when correction is made for the molecular orientation, there still remains a large difference in intrinsic 
birefringence among cellulose, xanthan, and schizophyllan. This difference may be ascribed to the difference in chain conformation among these glucans for the reason given below. Schizophyllan exists in water in the form of a triple helix consisting of loosely helical $\beta$-1,3D-glucan chains, ${ }^{8}$ whereas xanthan consists of $\beta$-1,4-D-glucan chains as cellulose and may have a more extended helical conformation. ${ }^{19,20}$ In the crystalline state, the virtual bond connecting the oxygen atoms attached to the 1 and 4 carbon atoms is in the direction of the maximum polarizability of the glucose residue $^{15}$ and inclined from the crystalline $\mathrm{c}$ axis by $66,30.9$, and $18.4^{\circ}$, for schizophyllan, ${ }^{21}$ xanthan, ${ }^{22}$ and cellulose, ${ }^{16}$ respectively. Thus it follows that the main chain glucose residues in schizophyllan make a small negative contribution to $n_{\alpha}-n_{\gamma}$. The side chain glucose residue of this polymer, about $1 / 3$ as heavy as the main repeating unit, cannot make a contribution larger than $0.082 / 4$. For xanthan, the contribution per main chain glucose residue was estimated to be about 0.05 . We suggest that the contribution from ionizable pendant groups, about twice the main chain weight, is large enough to offset this main chain contribution, yielding a negative $n_{\alpha}-n_{\gamma}$ as observed. Thus, we see in these two polysaccharides that the movable pendant groups play an equally important role as the main chain in determining $n_{\alpha}-n_{\gamma}$.

\section{Optical Rotatory Dispersion}

As noted in connection with Figure 5, eq 2 does not hold accurately over an extended range in wavelength. Indeed, for every case examined, the plot of $\Theta v s . \lambda^{-2}$ curved upward at shorter wavelengths and appeared to have a finite negative ordinate intercept. This is clearly seen in the data for the $14.34 \mathrm{wt} \%$ solution in the quartz cell shown in Figure 7. The data can be represented accurately by a simple Drude equation

$$
[\alpha]_{\lambda}=A \lambda_{0}^{2} /\left(\lambda^{2}-\lambda_{0}^{2}\right)
$$

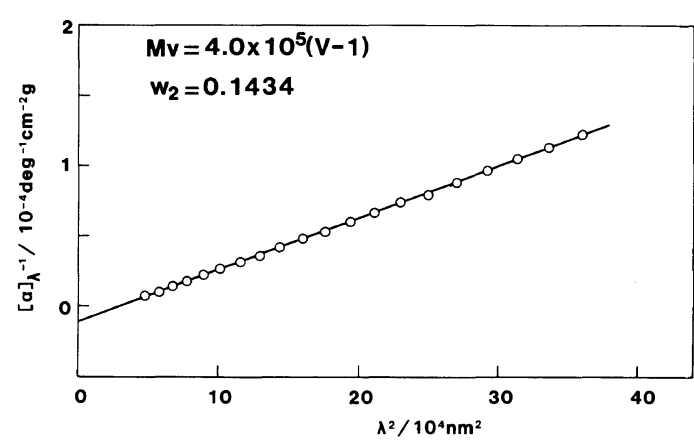

Figure 7. The ORD data for the cholesteric solution in Figure 3 reproduced in linear plots (see the text).

where $A$, and $\lambda_{0}$ are numerical constants. A similar deviation from the de Vries equation can be seen in the polypeptide data exhibiting a finite ordinate intercept, ${ }^{3,4,11}$ although the upward deviation is not appreciable because the data are limited to relatively larger wavelengths. On the other hand, the data for the isotropic solution shown in Figure 3 can be fitted accurately by an equation of the MoffittYang type. Patel and DuPré 7 have already remarked on these trends in their polypeptide data. Thus, we may conclude that an ORD curve of the type expressed by eq 7 is not due to the chiral nature of individual macromolecules but characteristic of the liquid crystal. No theoretical interpretation of this ORD behavior has as yet been given.

\section{Pretransitional Phenomena}

It was found in the previous study ${ }^{1}$ that at $25^{\circ} \mathrm{C}$, aqueous solutions of a schizophyllan sample of 478,000 molecular weight were biphasic at $w_{2}$ between 0.0965 and 0.128 . The solution with $w_{2}=0.0955$ was isotropic above $25^{\circ} \mathrm{C}$ but separated into two phases below $5^{\circ} \mathrm{C}$. ORD curves for this solution were measured as a function of temperature above $5^{\circ} \mathrm{C}$. The curves obtained above $10^{\circ} \mathrm{C}$ were essentially the same as the isotropic curve shown in Figure 3, but the optical rotatory power was remarkably enhanced when the temperature was lowered close to $5^{\circ} \mathrm{C}$. Figure 8 shows how 


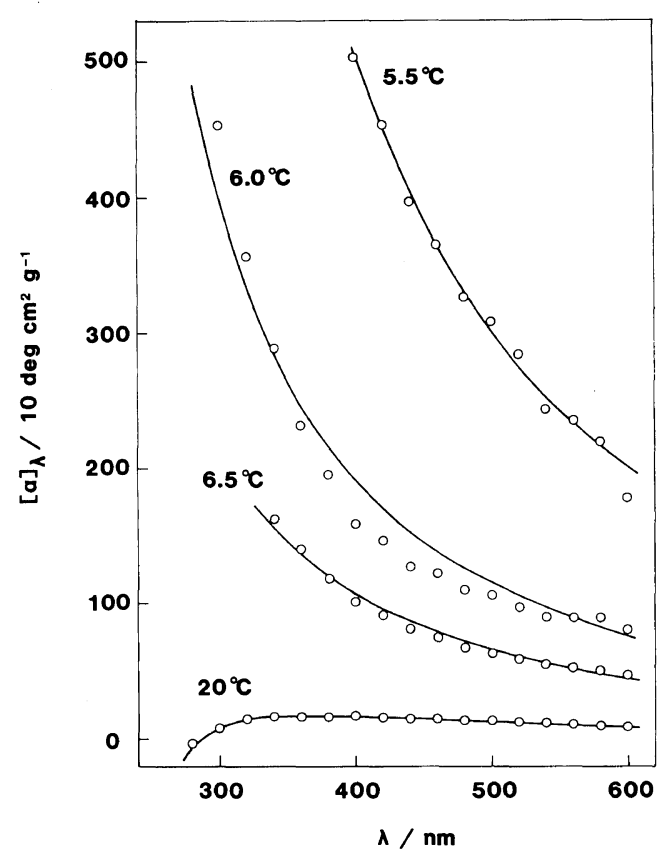

Figure 8. Temperature dependence of ORD curves of an isotropic solution of schizophyllan with $w_{2}=0.0955$ near the isotropic-biphasic boundary. Curve for $20^{\circ} \mathrm{C}$, the Moffitt equation; curves for $6.5,6.0$, and $5.5^{\circ} \mathrm{C}$, the Drude equation.

the ORD curve changes with lowering temperature. It can be seen that the curves at lower temperatures resemble the cholesteric curve in Figure 3 and that a transition from isotropic to cholesteric behavior occurs around $7^{\circ} \mathrm{C}$. The enhanced optical rotation may be attributed to embryonic cholesteric spherulites produced by concentration fluctuation in the solution, although no macroscopic phase separation occurred above $5.5^{\circ} \mathrm{C}$ within one $\mathrm{h}$ of observation. Thus, we see that the "pretransitional phenomenon" reported by Patel and DuPré for polypeptide liquid crystals also exists in our schizophyllan liquid crystals. This phenomenon will be persued in greater detail using ORD and light scattering techniques in a future study.

Acknowledgement. The authors wish to thank Dr. Yasuhiro Takahashi of our department for his comments on the conformations of schizophyllan, xanthan, and cellulose.

\section{REFERENCES}

1. K. Van, T. Norisuye, and A. Teramoto, Mol. Cryst. Liq. Cryst., 78, 123 (1981).

2. K. Van and A. Teramoto, Polym. J., 14, 999 (1982).

3. C. Robinson, Trans. Faraday Soc., 52, 571 (1956).

4. C. Robinson, Tetrahedron, 13, 219 (1961).

5. H. de Vries, Acta Cryst., 4, 219 (1951).

6. S. Chandrasekhar, "Liquid Crystals," Cambridge University Press, Cambridge, 1977, Chapter 4.

7. D. L. Patel and D. B. DuPré, J. Chem. Phys., 72, 2515 (1980).

8. T. Norisuye, T. Yanaki, and H. Fujita, J. Polym. Sci., Polym. Phys. Ed., 18, 547 (1980).

9. T. Itou, K. Van, and A. Teramoto, presented to the U.S.-Japan Joint Seminar on Polymer Liquid Crystals, Kyoto, 1983.

10. Y. Uematsu and I. Uematsu, Polym. Prepr., Div. Polym. Chem. Am. Chem. Soc., 20, 66 (1979).

11. H. Toriumi, Y. Kusumi, I. Uematsu, and Y. Uematsu, Polym. J., 11, 863 (1979).

12. So-Lan Tseng, A. Valente, and D. G. Gray, Macromolecules, 14, 715 (1981).

13. D. B. DuPré and F.-M. Lin, Mol. Cryst. Liq. Cryst., 75, 217 (1981).

14. G. Maret, M. Milas, and M. Rinaudo, Polym. Bull., 4, 291 (1981). These authors gave $0.025 \mathrm{~cm}^{3} \mathrm{~g}^{-1}$ for $|\Delta n| / c$, but we found a negative $\Delta n$ for the liquid crystal of aqueous xanthan.

15. P. H. Hermans, "Physics and Chemistry of Cellulose Fibers," Elsevier Publ., Amsterdam, 1948, p 23.

16. K. H. Gardner and J. Blackwell, Biopolymers, 13, 1975 (1974).

17. To better approximation, the right-hand side of eq 6 should be multiplied by a factor $\left(n_{\|}{ }^{2}+2\right)\left(n_{\perp}{ }^{2}+2\right)$ $\left(n_{\alpha}+n_{\gamma}\right) /\left[\left(n_{\alpha}{ }^{2}+2\right)\left(n_{\gamma}{ }^{2}+2\right)\left(n_{\|}+n_{\perp}\right)\right]$ which increases very gradually with increasing $\phi$ and approaches unity as $\phi$ tends to unity. In the $\phi$ range of interest, it is close to 0.9 .

18. M. Doi, J. Polym. Sci., Polym. Phys. Ed., 19, 229 (1981).

19. G. Holzwarth, Carbohydr. Res., 66, 173 (1978).

20. G. Paradossi and D. A. Brant, Macromolecules, 15, 874 (1982).

21. Y. Takahashi, private communication.

22. K. Okuyama, S. Arnott, R. Moorhouse, M. D. Walkinshaw, E. D. T. Atkins, CH. Wolf-Ullish, "Fiber Diffraction Methods;" A. D. French, K. H. Gardner, Ed., American Chemical Society, Washington, D.C., 1980, Chapter 26, ACS Sym. Ser., No. 141, p 411. 